\title{
Repurposing infectious disease vaccines for intratumoral immunotherapy
}

To cite: Melero I, Gato M, Shekarian T, et al. Repurposing infectious disease vaccines for intratumoral immunotherapy. Journal for ImmunoTherapy of Cancer 2020;8:e000443. doi:10.1136/jitc-2019-000443

Accepted 13 January 2020

\section{Check for updates}

(c) Author(s) (or their employer(s)) 2020. Re-use permitted under CC BY-NC. No commercial re-use. See rights and permissions. Published by BMJ.

\section{${ }^{1}$ Inmunology and}

Immunotherapy department, Centro de Investigación Médica Aplicada (CIMA) Avda Pio XII, 55 31008, Pamplona, Spain ${ }^{2}$ Centre de Recherche en Cancérologie de Lyon (CRCL), UMR INSERM U1052 CNRS 5286 Centre de Lutte contre le Cancer Léon Bérard, Université de Lyon, 69008, Lyon, France

${ }^{3}$ Gustave Roussy, INSERM U1015, Université Paris-Saclay, Drug Development Department (DITEP), Gustave Roussy, 94805, Paris, France

Correspondence to

Dr Ignacio Melero;

imelero@unav.es

\author{
Ignacio Melero, ${ }^{1}$ Maria Gato, ${ }^{1}$ Tala Shekarian, ${ }^{2}$ Angela Aznar, ${ }^{1}$ \\ Sandrine Valsesia-Wittmann (D) ,2 Christophe Caux, ${ }^{2}$ Iñaki Etxeberria, \\ Alvaro Teijeira, ${ }^{1}$ Aurelien Marabelle ${ }^{3}$
}

\begin{abstract}
Intratumoral delivery of viruses and virus-associated molecular patterns can achieve antitumor effects that are largely mediated by the elicitation or potentiation of immune responses against the malignancy. Attenuated vaccines are approved and marketed as good manufactiring practice (GMP)-manufactured agents whose administration might be able to induce such effects. Recent reports in mouse transplantable tumor models indicate that the rotavirus, influenza and yellow fever vaccines can be especially suitable to elicit powerful antitumor immunity against cancer following intratumoral administration. These results highlight that intratumoral anti-infectious vaccines can turn cold tumors into hot, and underscore the key role played by virusinduced type I interferon pathways to overcome resistance to immune checkpoint-targeted antibodies.
\end{abstract}

From Coley's clinical experiments with local treatment with bacteria or bacteria toxins ${ }^{1}$ to the generalized use of bacillusof Calmette and Guerin (BCG) for superficial bladder cancer, multiple lines of evidence suggest that local instigation of infectious micro-organisms or synthetic molecules mimicking their components, the so-called pathogen-associated molecular patterns (PAMPs) acting as pattern recognition receptor agonists (PRRs), can be beneficial against cancers. ${ }^{2}$

In the case of viruses, multiple attempts have been made to focus cytopathic effects against cancer, using the so-termed oncolytic viruses. Mounting evidence indicates that replicationcompetent oncolytic viruses and replicationdefective viral vectors exert their therapeutic effects mainly as a result of more potent antitumor immune responses. ${ }^{3}$ Such efficacy can be augmented when the genome of the virus is armed with genes that enhance immunity, such as cytokines and costimulatory factors. ${ }^{3}$

Intratumoral engineered variants of adenovirus, vaccinia virus, herpes simplex 1 virus (HSV), Newcastle disease virus and reovirus have shown remarkable activity in preclinical models and have progressed or are progressing to the clinic. The most advanced agent of this kind is T-vec (Talimogene laherparepvec), an attenuated HSV engineered to encode granulocyte-macrophage colony-stimulating factor (GM-CSF) which is approved for intratumoral infection in advanced melanoma ${ }^{4}$ and which seems to render synergistic effects if combined with systemic treatment with anticytotoxic T-lymphocyte antigen 4 (CTLA- 4$)^{5}$ or anti-programmed death-ligand 1 (PD1 $)^{6}$ monoclonal antibodies as immune checkpoint inhibitors.

In essence, the main factors that determine the antitumor effect are believed to be due to (1) immunogenic tumor cell death that releases antigens that can be presented by dendritic cells (especially cDC1 cells) and presentation of immunostimulatory molecules (calreticulin membrane expression, ATP release in the tumor microenvironment) ${ }^{7}$; (2) presence of moieties that are recognized by innate receptors of the immune system, triggering maturation of dendritic cells and causing local inflammation notably via type I interferon secretion ${ }^{7}$; and (3) recruitment and activation of antitumor T cells. ${ }^{7}$

In this scenario our group set out to identify routinely used attenuated viral vaccines that could be used via the intratumoral route. The advantage of repurposing such approved and marketed agents is that clinical development would be much simplified based on solid safety records.

Rotavirus infection is a serious threat causing severe diarrhea in infants. Attenuated vaccines are protective when orally given. These commercially available double-stranded RNA (dsRNA) viral attenuated strains turned out to be very potent stimulators of the nuclear factor kappa-light-chain-enhancer of activated B cells $(\mathrm{NF \kappa B})$ and type I interferon pathways. Interestingly, this stimulation is independent from the innate Toll-like immune receptors but dependent on another PRRs known as retinoic acid induced gene 1 (RIG-I), which is able to detect intracytoplasmic dsRNA. ${ }^{8}$ Furthermore, rotavirus exerts cytopathic effects, killing a 
variety of both adult and pediatric cancer cell lines in culture with features of immunogenic cell death (ATP release). ${ }^{7}$ Interestingly, if given intratumorally to mouse bearing transplantable tumors, including pediatric syngeneic neuroblastoma murine models, remarkable local therapeutic effects are elicited that are dependent on natural killer cells (NK), CD4 and CD8 T cell immunity. Most importantly, in murine models that are refractory to immune checkpoint-targeted therapies, intratumoral rotavirus is able to synergize and overcome resistance to anti-CTLA-4 or anti-PD-L1 monoclonal antibodies, including against tumor lesions growing subcutaneously on the contralateral uninjected flank (anenestic effect).9 It is of important note that prevaccination of mice prior such intratumoral virotherapy does not spoil efficacy.

In the same line, the yellow fever vaccine strain 17D used for travelers and dwellers in endemic areas is also cytopathic for a large panel of human and mouse tumor cell lines, and intratumor administration clearly delays tumor progression in a manner mediated by CD8 T cell immunity with some measurable effect against non-injected concomitant tumors. ${ }^{10}$ Very importantly, efficacy was potentiated by previous vaccination against the virus in a manner dependent on $\mathrm{T}$ cell antiviral immunity. In this case additive effects with systemic immunostimulatory monoclonal antibodies directed to anti-PD1 or anti-CD137 were found.

Similarly, Newman et al reported that intratumoral injections of anti-influenza vaccines could also elicit immunemediated antitumor activity in an aggressive melanoma syngeneic transplantable tumor model (Proceedings of the National Academy of Sciences (PNAS), in press). Similar to the rotavirus observation, inactivated influenza virus was sufficient to show activity against tumors on intratumoral administration. However, and most surprisingly, only unadjuvanted inactivated influenza vaccines were able to generate such antitumor efficacy. Indeed, squalene-based adjuvanted influenza vaccines were losing their antitumor activity because adjuvants were recruiting interleukin-10-secreting B regulatory cells.

Importantly, for none of these three strategies, preexisting immunization against the corresponding pathogen/vaccine did preclude the antitumor efficacy of its intratumoral delivery.

It is to be considered that while 17D yellow fever and influenza vaccines are approved for injected routes of administration, rotavirus vaccines are only approved for oral administration. However, because rotavirus vaccines have mostly polysaccharides as adjuvants, they should, like for yellow fever and influenza vaccines, be amenable to a rapid clinical translation for in situ virus-vaccine cancer immunotherapy.

All considered, these new studies open the field for repurposing viral vaccines in strategies combining local virotherapy and other immunotherapy agents (figure 1 ). This is not at odds with intratumoral compounds based on viral immunity such as Toll-like receptor (TLR3, TLR9) or stimulator of interferon genes protein (STING) agonists that are being developed for intratumoral administration. ${ }^{12}$ In any case, it is clear that what best mimics a local viral infection is the virus itself. These agents have a number of pros and cons if compared with the preclinically studied repurposed vaccines. Prevaccination is probably an interesting safety feature that seems to increase efficacy or that at least does not spoil it. Another question that can only be addressed in humans is the prime-boosting effect of injecting multiple tumor lesions at different time points within the same patient. Such strategy could better address the cancer heterogeneity existing within metastatic patients while amplifying pre-existing anticancer immune responses. Also, these intratumoral virus vaccines could be

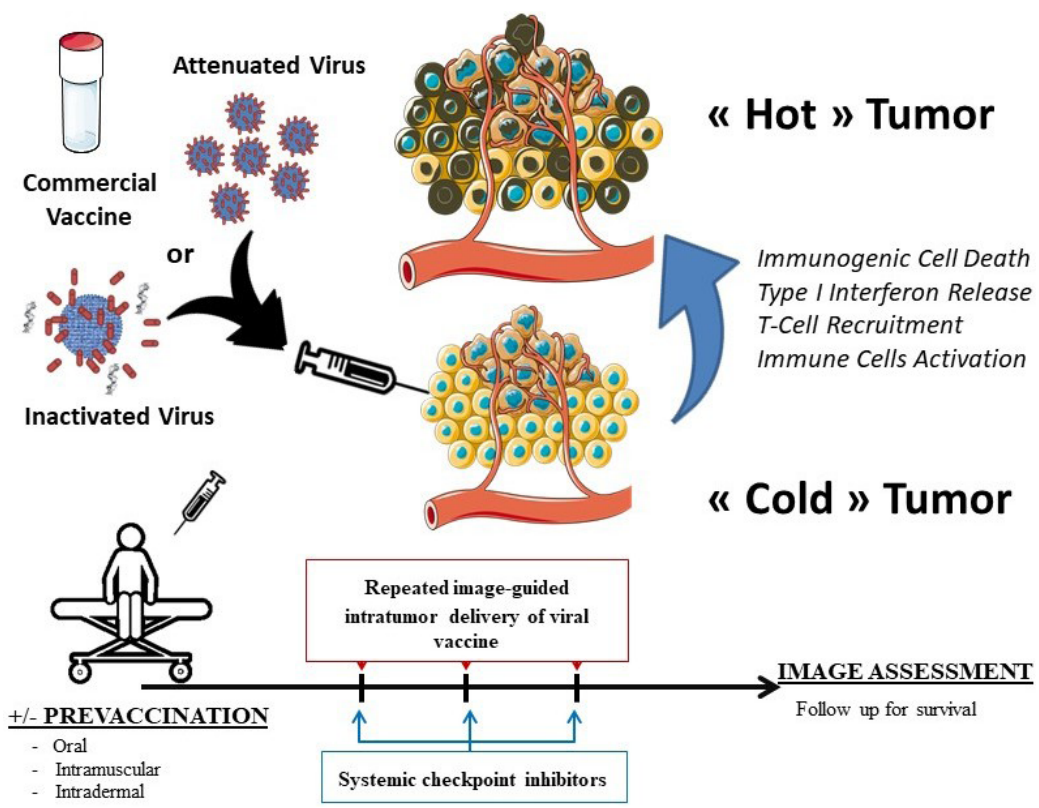

Figure 1 Repurposing anti-infectious viral vaccines for intratumoral immunotherapy to turn cold tumors into hot and overcome resistance to immune checkpoint-targeted therapies. 
of great value to trigger the antitumor immunity in a neoadjuvant setting in order to avoid postsurgery relapses. Last but not least, these commercially available and pediatricgrade anti-infectious vaccines could be of interest to treat cancers arising in infants and children, or any other rare tumor indication which is not frequent enough to benefit from registration trials of pattern recognition receptor agonists and oncolytic viruses.

Twitter Sandrine Valsesia-Wittmann @SVW

Contributors IM and AM wrote the manuscript. All authors revised and discussed the content.

Funding This project was supported by MINECO SAF2017-83267-C2-1-R (AEI/ FEDER, UE).

Competing interests Consulting: BMS, AZ, Roche, F-star, Genmab, Alligator, Bioncotech, Numab, EMD. Grants: Roche, BMS, Alligator, Bioncotech.

Patient consent for publication Not required.

Provenance and peer review Commissioned; externally peer reviewed.

Open access This is an open access article distributed in accordance with the Creative Commons Attribution Non Commercial (CC BY-NC 4.0) license, which permits others to distribute, remix, adapt, build upon this work non-commercially, and license their derivative works on different terms, provided the original work is properly cited, appropriate credit is given, any changes made indicated, and the use is non-commercial. See http://creativecommons.org/licenses/by-nc/4.0/.
Sandrine Valsesia-Wittmann http://orcid.org/0000-0001-8764-0825

\section{REFERENCES}

1 Aznar MA, Tinari N, Rullán AJ, et al. Intratumoral delivery of Immunotherapy-Act locally, think globally. J Immunol 2017;198:31-9.

2 Vanpouille-Box C, Hoffmann JA, Galluzzi L. Pharmacological modulation of nucleic acid sensors - therapeutic potential and persisting obstacles. Nat Rev Drug Discov 2019;18:845-67.

3 Harrington K, Freeman DJ, Kelly B, et al. Optimizing oncolytic virotherapy in cancer treatment. Nat Rev Drug Discov 2019;18:689-706.

4 Andtbacka RHI, Kaufman HL, Collichio F, et al. Talimogene laherparepvec improves durable response rate in patients with advanced melanoma. JCO 2015;33:2780-8.

5 Puzanov I, Milhem MM, Minor D, et al. Talimogene Laherparepvec in combination with ipilimumab in previously untreated, unresectable stage IIIB-IV melanoma. J Clin Oncol 2016;34:2619-26.

6 Ribas A, Dummer R, Puzanov I, et al. Oncolytic virotherapy promotes intratumoral T cell infiltration and improves anti-PD-1 immunotherapy. Cell 2017:170:1109-19.

7 Galluzzi L, Buqué A, Kepp O, et al. Immunogenic cell death in cancer and infectious disease. Nat Rev Immunol . 2017;17:97-111 https:// doi.org/

8 Shekarian T, Sivado E, Jallas A-C, et al. Repurposing rotavirus vaccines for intratumoral immunotherapy can overcome resistance to immune checkpoint blockade. Sci Transl Med 2019;11:eaat5025.

9 Marabelle A, Andtbacka R, Harrington K, et al. Starting the fight in the tumor: expert recommendations for the development of human intratumoral immunotherapy (HIT-IT). Ann Oncol 2018;29:2163-74.

10 Aznar MA, Molina C, Teijeira A, et al. Repurposing the yellow fever vaccine for intratumoral immunotherapy. EMBO Mol Med 2020;12:e10375. 